\title{
Stimulus generalization of an illness-induced aversion to different intensities of colored water in Japanese quail
}

\author{
JAMES A. CZAPLICKI, DAVID E. BORREBACH, and HARDY C. WILCOXON \\ George Peabody College for Teachers, Nashville, Tennessee 37203
}

\begin{abstract}
After quail (Coturnix coturnix japonica) experienced a toxicosis following ingestion of water colored dark blue or light blue, the stimulus generalization of the aversion to light, medium, and dark blue water was investigated. Birds made ill following ingestion of dark blue water demonstrated a reduced aversion when tested with intensities of blue water weaker than the conditioning intensity. Birds made ill following ingestion of light blue water showed stronger aversions as a function of increasing test intensity, suggesting a stimulus intensity dynamism effect. The stimulus generalization and stimulus-intensity dynamism effects observed in this study where visual cues were used is in agreement with other results within the illness-induced aversions paradigm where rats were tested with varying concentrations of a gustatory stimulus.
\end{abstract}

Stimulus generalization is said to occur when a reaction that has been learned to a particular stimulus is now elicited by stimuli similar to the one used during conditioning. In the ever changing environment in which organisms live, the adaptive value of stimulus generalization is obvious since a stimulus exactly like the one used during conditioning is not likely to occur again. While the phenomenon of stimulus generalization has been extensively studied in many learning situations, few examples of generalization exist within the illness induced aversions paradigm.

Nachman (1962) concluded that lithium chloride $(\mathrm{LiCl})$ and sodium chloride $(\mathrm{NaCl})$ tasted similar to the rat because adrenalectomized rats exhibited no preference between equimolar solutions of $\mathrm{LiCl}$ and $\mathrm{NaCl}$, even though $\mathrm{LiCl}$ is known to produce illness in rats. In a subsequent study Nachman (1963) found that rats made ill by consumption of $\mathrm{LiCl}$ demonstrated the greatest aversion to both $\mathrm{LiCl}$ and $\mathrm{NaCl}$ and a smaller aversion to ammonium chloride, with potassium chloride being the least noxious salt. Furthermore, this gradient of stimulus generalization to different salts paralleled independently obtained recordings from the chorda tympani nerve of the rat.

Other examples of generalization along the dimension of taste quality are also available. Roll (reported by Smith, 1971) found that a radiation-induced aversion to sucrose generalized to a glucose solution but not to a maltose solution. Smith (1971) demonstrated that a radiation-induced aversion to a glucose and saccharin solution generalized to sacchain-only solutions and possibly to glucose-only solutions (to a lesser extent). It has also been found that rats demonstrating a radiation induced aversion to DL-alanine, glycine or sodium saccharin generalized their aversions within this class of stimuli, so that if made ill on any one of these stimuli,

This research was supported by NICHD Grant 00973. Requests for reprints should be sent to Hardy C. Wilcoxon, Department of Psychology, Box 512, George Peabody College for Teachers, Nashville, Tennessee 37203. they showed an aversion to the other two. The rats did not, however, generalize their aversion to all novel solutions, as they did not avoid D-glucose or potassium chloride solutions (Tapper \& Halpem, 1968).

Besides the above studies dealing with generalization along the dimension of taste quality, several experiments have been concerned with generalization along the intensity continuum. Tapper and Halpern (1968) demonstrated that rats that had received sodium saccharin or DL-alanine paired with radiation generalized their aversions to weaker concentrations so that the weaker the intensity (relative to the conditioning intensity), the less pronounced was the aversion. Recently, in a study where rats were tested with intensities of a $\mathrm{NaCl}$ solution that were either higher or lower than the conditioning intensity of $\mathrm{NaCl}$, Nowlis (1974) found a gradient of stimulus generalization wherein the more intense the gustatory and/or olfactory stimulus presented during testing, the more pronounced was the suppression of drinking in the presence of that stimulus.

Generalization of illness-induced aversions has also been noted in garter snakes. Burghardt, Wilcoxon, and Czaplicki (1973) observed that garter snakes that had experienced a delayed toxicosis following their ingestion of night crawlers subsequently demonstrated an aversion to an extract prepared from the surface substances of night crawlers (presumably a less intense version of the conditioning stimulus). This aversion to night crawler extract (relative to minnow extract) was probably mediated by olfactory cues.

We know of no experiments which demonstrate stimulus generalization of an illness-induced aversion mediated by a sensory modality other than gustation or olfaction. It appears, however, that illness-induced aversions may also be mediated by visual cues under certain conditions. Brower (1969) demonstrated that blue jays are able to learn to avoid toxic monarch butterflies on the basis of purely visual cues. Brower maintained that the blue jays associated the taste of the 
monarchs with illness when the taste was reinstated during regurgitation and then associated the visual cues with the taste when the birds next encountered the butterflies. In a similar vein, Wilcoxon, Dragoin, and Kral (1971) found that quail that had experienced a delayed toxicosis following ingestion of colored water subsequently showed an aversion to the colored water during extinction tests. In a recent study, Braveman (1974) found that guinea pigs may also be able to associate visual cues with a delayed illness.

Given that illness-induced aversions are capable of being mediated by visual cues, it is quite possible that a type of stimulus generalization similar to that observed when olfactory and/or gustatory cues mediate the aversion might also be observed when visual cues are involved. This experiment was designed to ascertain the nature of the stimulus generalization gradient along the continuum of intensity produced in quail following an aversive conditioning procedure in which the birds experienced an illness (intraperitoneal injection of $\mathrm{LiCl}$ ) subsequent to their ingestion of a colored fluid.

\section{Subjects}

The subjects were 60 adult male quail (Coturnix coturnix japonica). They were individually housed in wire cages (15 x 51 $x 23 \mathrm{~cm}$ high) and allowed free access to food (Purina bird chow) throughout the course of the experiment. The bird housing room was illuminated by ceiling mounted fluorescent lights between 0400 and $1800 \mathrm{~h}$. The temperature in the housing room was maintained at $25^{\circ} \mathrm{C}( \pm 2)$.

\section{Procedure}

Prior to the beginning of the experimental regimen, the birds were trained over a 5-day period to drink all of their daily water from 30-ml glass Richter tubes. The Richter tubes were clipped to the front of the birds' housing cages at the beginning of each daily drinking session and removed when the session was terminated. Distilled water was presented at $0930 \mathrm{~h}$ each day, and the time allowed for drinking was reduced to a 10 -min daily drinking period.

All birds then received 3 days of preexposure to blue water in order to counteract any neophobic tendencies that might otherwise be present later on treatment day. The three intensities of blue water which were to be used in the main experimental treatment and tests were used in the preexposure period. On the first day, half of the birds received dark blue water $(5.0 \mathrm{cc}$ commercial food coloring per liter of distilled water) and half received light blue water $(.5 \mathrm{cc} / 1)$. On Day 2 , all birds received medium blue water $(1.5 \mathrm{cc} / 1)$, and on Day 3 the conditions of Day 1 were reversed. Whether a bird received dark or light blue water first was determined at random, and all preexposures were given during the daily 10 -min drirising periods.

For the next 8 days, all birds received plain distilled drinking water during their daily drinking periods so as to provide them with a stable background of experience against which the reintroduction of blue water would be relatively novel on treatment day.

The birds were then randomly divided into five groups and five treatment day conditions were imposed that provided two conditioning groups and three control groups. One conditioning group (dark-LiCl) received dark blue water followed, $30 \mathrm{~min}$ later, by an intraperitoneal injection of a .15-molar LiCl solution at a ratio of $3 \%$ liquid volume per $100 \mathrm{~g}$ body weight. The second conditioning group (light-LiCl) received light blue water before receiving the toxic injection. Corresponding control groups (dark-saline, and light-saline) drank either dark or light blue water followed, 30 min later, by a similar injection of normal saline. The fifth group was a toxicosis control group (plain-LiCl). It received plain distilled water on treatment day followed, $30 \mathrm{~min}$ later, by the same toxic injection that the conditioning groups received. Following treatment day, all birds were allowed 2 days of drinking on plain water. Subsequent to this 2-day recovery period, extinction tests began.

On Extinction Day $1\left(\mathrm{E}_{1}\right)$, all subjects were tested with a two-bottle test in which plain water and the conditioning intensity of blue were simultaneously present, with the exception of the plain-LiCl birds who were presented with plain water and light blue water.

On Extinction Day $2\left(\mathrm{E}_{2}\right)$, all subjects were tested with a two-bottle test in which light blue water and dark blue water were offered simultaneously.

On Extinction Day $3\left(\mathrm{E}_{3}\right)$, all subjects received a three-bottle test in which light, medium, and dark blue water were offered simultaneously. During the three-bottle tests, light, medium, and dark blue water were offered simultaneously by affixing the three tubes to the birds' cages during their 10-min drinking sessions. The arrangement of the three bottles on each bird's cage was (from left to right) either light-medium-dark or darkmedium-light, with the two arrangements counterbalanced within groups.

On Extinction Day $4\left(\mathrm{E}_{4}\right)$, all subjects were tested with the procedure described for $E_{1}$.

Seven subjects died during the course of this experiment: two each from the light- $\mathrm{LiCl}$ and plain- $\mathrm{LiCl}$ groups, and one each from the remaining three groups. For purposes of analysis, preference scores were used. On $E_{1}$ and $E_{4}$, blue preference scores and plain preference scores were calculated. Blue preference scores were calculated by dividing the quantity (in ml) of blue water drunk by the total quantity of fluid (plain and blue) drunk and the plain preference scores were calculated by dividing the quantity of plain water drunk by the total fluid (plain and blue) drunk. A blue or plain preference score of .50 indicates that half of the total fluid consumed was blue or plain respectively; a blue or plain preference score of 1.00 indicates that all of the fluid consumed was blue or plain respectively, and so on. On $E_{2}$ and $E_{3}$, preference scores were calculated by dividing the given intensity of blue water consumed by the total fluid (light blue and dark blue on $\mathrm{E}_{2}$, and light, medium, and dark blue on $E_{3}$ ) drunk for that day. For any given individual extinction test, then, the sum of the preference scores was equal to 1.00 .

\section{RESULTS}

The results of this experiment are depicted in Figure 1. When quail were tested with intensities of blue water weaker than the conditioning intensity, their aversions were less pronounced. When tested with intensities higher than the conditioning intensity, the quail demonstrated increasingly stronger aversions.

On $E_{1}$ and $E_{4}$, the subjects were tested with two-bottle tests in which plain water and the conditioning intensity of blue water were offered simultaneously. An analysis of variance (Drug by Intensity by Days) utilizing the blue preference scores of the light-LiCl, light-saline, dark-LiCl, and dark-saline subjects, revealed a main drug effect $(F=19.99$; df $=1 / 39 ; p<.01)$ wherein the light- $\mathrm{LiCl}$ and the dark- $\mathrm{LiCl}$ subjects showed an aversion to blue water. Subsequent statistical comparisons revealed that, on both $\mathrm{E}_{1}$ and $\mathrm{E}_{4}$, the dark- $\mathrm{LiCl}$ subjects demonstrated a significant aversion to dark blue 
Figure 1. Mean preference scores for fluids drunk by each of the five experimental groups during extinction days. $(P=$ plain water, $\mathbf{L}=$ light blue water, $\mathbf{M}=$ medium blue water, $D=$ dark blue water.) Preference scores were obtained for individual subjects by dividing the amount of a particular fluid drunk by the total fluid intake for the given animal during a drinking session.

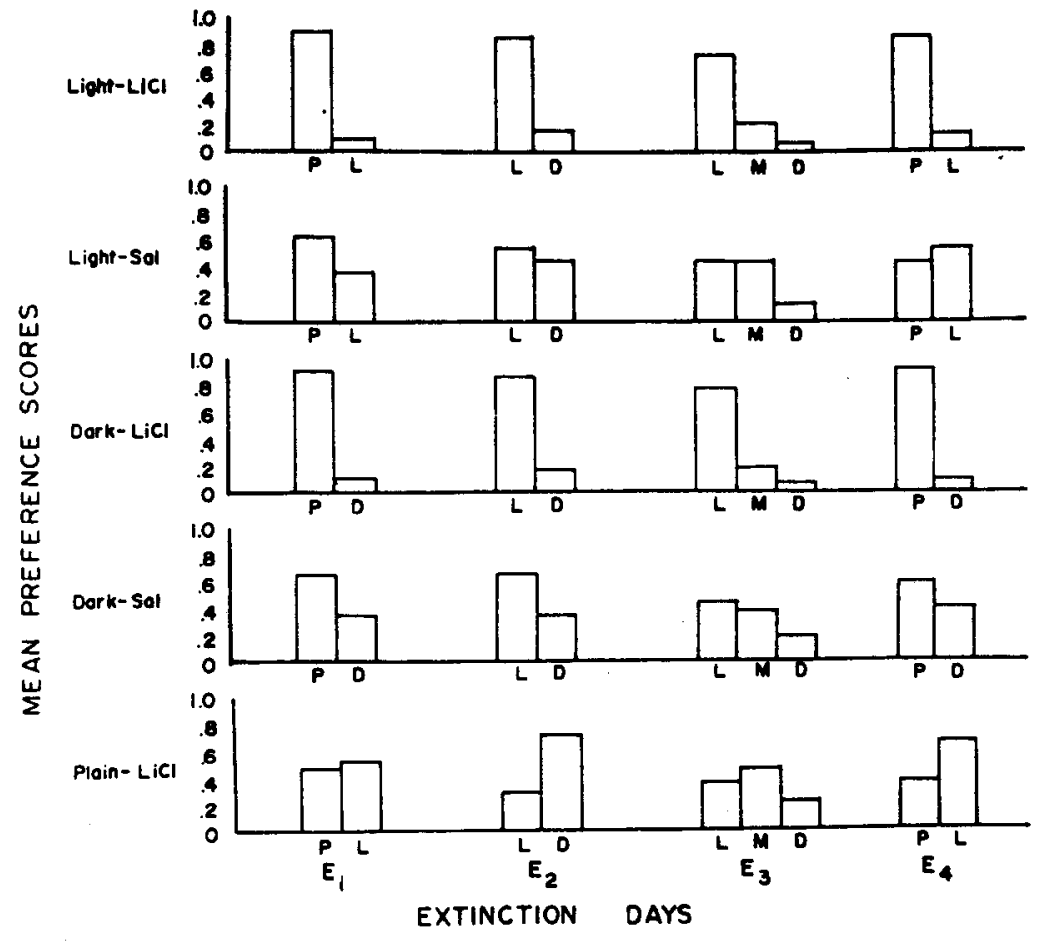

water when compared to the dark-saline subjects, while the light- $\mathrm{LiCl}$ birds showed a significant aversion to light blue water when compared to the light-saline subjects. The subjects in the two groups that received either light blue or dark blue water on the treatment day, followed by an injection of $\mathrm{LiCl}$, demonstrated an aversion to that intensity of blue water when it was offered simultaneously with plain water.

On $E_{2}$, the birds were tested with a two-bottle test in which dark blue and light blue water were offered simultaneously. An analysis of variance (Drug by Intensity) using light blue preference scores revealed a main drug effect $(F=5.06 ; \mathrm{df}=1 / 39) ; \mathrm{p}<.05)$ wherein the light- $\mathrm{LiCl}$ and dark- $\mathrm{LiCl}$ subjects demonstrated a preference for light blue water. The light- $\mathrm{LiCl}$ subjects demonstrated a significant preference for light blue water when compared to the light-saline birds, while no significant difference was noted between the dark- $\mathrm{LiCl}$ and dark-saline groups. This failure to find a significant difference between the dark-saline and dark- $\mathrm{LiCl}$ groups is probably due to the unusually high preference for light blue water that the dark-saline birds exhibited on this day.

On $E_{3}$, all birds were tested with three-bottle tests in which light, medium, and dark blue water were offered simultaneously. An analysis of variance (Drug by Intensity) using light blue preference scores revealed a main drug effect $(F=5.06 ; \mathrm{df}=1 / 39 ; p<.05)$ wherein $\mathrm{p}<.01$ ) wherein the light- $\mathrm{LiCl}$ and dark-LiCl birds demonstrated a preference for light blue water. Subsequent comparisons revealed that the light-LiCl birds showed a significant preference for light blue water when compared to the light-saline birds, and the
dark-LiCl birds showed a significant preference for light blue water when compared to the dark-saline birds.

Furthermore, the effects observed in the two conditioning groups were not due to a toxicosis induced neophopia as the toxicosis group (plain-LiCl) did not drink in a manner similar to the light- $\mathrm{LiCl}$ or dark- $\mathrm{LiCl}$ groups (see Figure 1). Statistical tests using blue preference scores indicated that the plain- $\mathrm{LiCl}$ group was significantly different from the light- $\mathrm{LiCl}$ group on $\mathrm{E}_{1}$ and $E_{4}(t=3.03$, df $=18, p<.01 ; t=3.35$, df $=18$, $p<.01$, respectively). On $E_{2}$ and $E_{3}$, when light blue preference scores were utilized, the plain- $\mathrm{LiCl}$ group was significantly different from the light- $\mathrm{LiCl}$ group $(\mathrm{t}=3.74, \mathrm{df}=18, \mathrm{p}<.01 ; \mathrm{t}=3.65, \mathrm{df}=18, \mathrm{p}<.01)$. Similar analyses revealed that at no time were the plain-LiCl birds statistically different from the this experiment were not due to a drug induced neophobia.

It should also be noted that the experiment reported here has been replicated on two other occasions in our laboratory with slightly different procedures each time. Briefly, the results obtained in this experiment seem to be reliable, since they were confirmed using both male and female quail, two or three injections, differing dosages of $\mathrm{LiCl}$, with and without a preexposure to blue period, and with different intensities for the three shades of blue.

\section{DISCUSSION}

Three aspects of the present findings deserve particular discussion: (1) generalization gradients to visual cues light-saline birds. Therefore, the gradients observed in 
were observed which are similar to other findings within the illness-induced aversions paradigm when gustatory cues were used; (2) the generalization gradients were influenced by a stimulus-intensity dynamism effect which was also similar to other results within the illness-induced aversions paradigm when gustatory cues mediated the aversion; (3) despite a 3-day preexposure period, statistically significant aversions were noted.

It appears that the relevant cues which become associated with a toxicosis are the cues, whether gustatory, olfactory, or visual, that an animal uses in its normal feeding behavior. Earlier work within the illness induced aversions paradigm indicated that bobwhite quail are able to demonstrate an aversion to both visual and gustatory cues, but that visual cues appear to be more salient (Wilcoxon et al., 1971). In view to this, it is not surprising that quail, birds which use visual cues in their normal foraging and feeding behavior and are also able to form an illness-induced aversion to these visual cues, demonstrate a gradient of stimulus generalization to different intensities of colored water that resembles similar gradients along the dimension of intensity observed when gustatory and/or olfactory cues mediate an aversion (Nowlis, 1974; Tapper \& Halpern, 1968).

A stimulus-intensity dynamism effect was also noted in this experiment wherein the strength of the aversion to blue water was a positive function of the testing intensity. A similar finding was noted by Nowlis (1974) when rats were tested with varying concentrations of a $\mathrm{NaCl}$ solution. It appears, then, that besides being present during more traditional classical conditioning experiments (Razran, 1949), stimulus-intensity dynamism effects also exist within the illness-induced aversions paradigm.

Another interesting finding observed in this experiment was the apparent lack of deleterious effects upon conditioning produced by the preexposure to blue period. Numerous experiments have found an attenuation in the strength of illness-induced aversions as a function of the animals having received preexposure to the conditioning stimulus (Elkins, 1973). Kalat and Rozin (1973) found that a single preexposure to casein hydrolysate resulted in a significant attenuation of the subsequent aversion to that solution even when 20 days of plain water drinking were interpolated between preexposure and conditioning trials. The quail in this experiment received 3 days of preexposure to the various intensities of blue water, one each of light, medium, and dark, and yet a significant aversion to the conditioning intensity was noted only on $E_{1}$ and $E_{4}$.

In several unreported experiments conducted in our laboratory, using quail as subjects, preexposure to the visual conditioning stimulus was used in an effort to reduce neophobia and thereby insure drinking of the visual stimulus on treatment days. Strong aversions were still noted. It is possible that visual cues are less salient than gustatory cues, and are also subject to more interference as the quail typically see a host of colors in the course of everyday maintenance operations (experiment er's clothes, etc.) Further experimentation will be required to ascertain the reasons for this apparent reduction in the typically observed deleterious effects of preexposure.

\section{REFERENCES}

Braveman, N. S. Poison-based avoidance learning with flavored or colored water in guinea pigs. Learning and Motivation, $1974,5,182-194$.

Brower, L. P. Ecological chemistry. Scientific American, 1969. 220 (2), 22-29.

Burghardt, G. M., Wilcoxon, H. C., \& Czaplicki, J. A. Conditioning in garter snakes: Aversion to palatable prey induced by delayed illness. Animal Learning \& Behavior, 1973, 1, 317-320.

Elkins, R. L. Attenuation of drug-induced bait shyness to a palatable solution as an increasing function of its availability prior to conditioning. Behavioral Biology, 1973, 9, 221-226.

Kalat, J. W., \& Rozin, P. "Learned safety"' as a mechanism in long-delay taste-aversion learning in the rat. Journal of Comparative and Physiological Psychology, 1973, 83, 198-207.

Nachman, $\dot{M}$. Taste preferences for sodium salts by adrenalectomized rats. Journal of Comparative and Physiological Psychology, 1962, 55, 1124-1129.

Nachman, $M$. Learned aversion to the taste of lithium chloride and generalization to other salts. Journal of Comparative and Physiological Psychology, 1963, 56, 343-349.

Nowlis, G. H. Conditioned stimulus intensity and acquired alimentary aversions in the rat. Journal of Comparative and Physiological Psychology, 1974, 86, 1173-1184.

Razran, G. Stimulus generalization of conditioned responses. Psychological Bulletin, 1949, 46, 337-367.

Smith, J. C. Radiation: Its detection and its effects on taste preferences. In E. Stellar and J. M. Sprague (Eds.), Progress in physiological psychology (Vol. 4). New York: Academic Press, 1971, pp. 53-118.

Tapper, D. N., \& Halpern, B. P. Taste stimuli: A behavioral categorization. Science, 1968, 161, 708-709.

Wilcoxon, H. C., Dragoin, N. B., \& Kral, P. A. nlness-induced aversions in rat and quail: Relative salience of visual and gustatory cues. Science, 1971, 171, 826-828.

(Received for publication Octobex 23, 1974 . Revision received July 21, 1975.) 